\title{
Conocimiento didáctico del contenido. Mis últimas investigaciones: CDC en lo afectivo, sobre la estequiometría y la indagación
}

\author{
Andoni Garritz
}

Artículo recibido: 08-05-2011 y aprobado: 15-07-2011

Pedagogical content knowledge. My last researches: PCK in the affective domain, on Stoichiometry and Inquiry

Resumen: En este artículo reúno varios temas recientemente investigados sobre el Conocimiento Didáctico del Contenido (CDC), aunque todavía alguno no ha sido concluido todavía. Para empezar, abordaré el espinoso tema de la afectividad y si forma parte o no del CDC; inmediatamente un tema de la enseñanza de la química, la estequiometría o ciencia de los cálculos químicos, que, por lo general, se emplea con un objetivo algorítmico de resolución de problemas, aunque puede dársele un mayor potencial; finalmente algo sobre el tema del CDC de la indagación, un tópico de reciente y amplio interés en la comunidad de educadores e investigadores, sobre todo desde su aparición en los Estándares Nacionales de la Educación Científica en los Estados Unidos.
Abstract: I have taken several recent researches on pedagogical content knowledge (PCK) to write this paper, although some of them are in course by now. To start with I will board the thorny topic of affectivity and if it can be included inside PCK or not; immediately a topic on chemistry education, Stoichiometry or the science of chemical calculations which, generally, it is used with an algorithmic problem solving aim, but it can be used with greater potential; finally some on the topic of inquiry, one that has acquire a lot of relevance in the literature, mainly because of its inclusion in the National Standards of Science Education in USA.
Palabras-clave: Conocimiento Didáctico del Contenido, afectividad, estequiometría, indagación.
Key words: Pedagogical Content Knowledge, affectivity, Stoichiometry, inquiry.

Facultad de Química, Universidad Nacional Autónoma de MéxicoCiudad Universitaria, Av. Universidad \# 300004510, México, D.F. MÉXICO. 


\section{Pedagogical content knowledge}

Nos cuenta Lee Shulman (1999) -en el prólogo de la obra editada por Julie Ges-Newsome y Norman Ledermancuando en el verano de 1983 dictó una conferencia en la Universidad de Texas, en Austin, la que tituló "El paradigma perdido en la investigación sobre la enseñanza":

Para mi delicia, el título aparentemente había estimulado discusiones serias entre los participantes, en anticipación a mi charla. Ellos se preguntaban: ¿Qué es lo que Shulman trae en mente como "el paradigma perdido"? Abundaban las especulaciones. Muchos predecían que yo identificaría como tal a la "cognición del profesor"... Otros nominaban al "contexto". Todavía algunos más especulaban que sería "la personalidad del profesor"... la mayoría recibió un fuerte impacto cuando declaré que el paradigma perdido era el estudio del contenido de la materia y su interacción con la pedagogía.

Shulman (1999: P. ix).

Esta propuesta sorprendió a todo mundo ya que hasta entonces los estudios sobre la enseñanza se habían enfocado en las formas de comportamiento del profesor más que en las de su pensamiento y sus conocimientos y creencias. Shulman (1987) nos dice que:

... al centrar nuestra atención en los tipos de conocimientos y destrezas necesarios para enseñar bien materias exigentes; es decir, al concentrarnos en la enseñanza de temas específicos - Huckleberry Finn, ecuaciones de segundo grado, el subcon- tinente indio, fotosíntesis - nos enteramos de la manera en que determinados tipos de conocimiento de la materia y las estrategias didácticas interactuaban en la mente de los profesores.

Shulman

(1987, traducción de 2005, p. 7)

De esta forma, Shulman desemboca en el 'Conocimiento base para la enseñanza' y, dentro de éste al Conocimiento Didáctico del Contenido ${ }^{1}$. Nos dice Shulman acerca de la gran importancia que tiene el conocimiento de la disciplina, pero también la forma de representarlo como algo crucial:

Un profesor sabe algo que otros no comprenden, presuntamente los alumnos. El profesor puede transformar la comprensión, las habilidades para desenvolverse, las actitudes o los valores deseados, en representaciones y acciones pedagógicas. Se trata de formas de expresar, exponer, escenificar o de representar de otra manera ideas, de suerte que los que no saben puedan llegar a saber, los que no entienden puedan comprender $y$ discernir, y los inexpertos puedan convertirse en expertos. Así pues, el proceso de enseñanza se inicia necesariamente en una circunstancia en que el profesor comprende aquello que se ha de aprender y cómo se lo debe enseñar.

Shulman

(1987, traducción de 2005, p. 9)

¿Cómo lograr la representación del contenido para que ésta sea inteligible, verosímil y fructífera para los estudiantes? (utilizamos aquí las características que Posner et al., en 1982,

\footnotetext{
${ }^{1}$ Nos referimos al Conocimiento Pedagógico del Contenido, como debe traducirse el "Pedagogical Content Knowledge" de Shulman y que, por respeto a las costumbres en España y Colombia, le llamaremos aquí como " Conocimiento Didactico del Contenido".
} 
mencionan como necesarias para el cambio conceptual). O como preguntaba Klafki a los profesores para preparar adecuadamente una clase (1958, p.45):

¿Qué fenómeno básico o principio fundamental, qué ley criterio, problema, método técnica o actitud puede captarse para ver el contenido como ejemplo.

¿Qué hechos, fenómenos, situaciones, experimentos, controversias, etc.; qué intuiciones son apropiadas para inducir a los estudiantes a preguntarse cuestiones dirigidas hacia la esencia y estructura del contenido en cuestión?

¿Qué ilustraciones, sugerencias, situaciones, observaciones, explicaciones, experimentos o modelos son apropiados para ayudar a los alumnos a responder, tan independientemente como sea posible, sus preguntas dirigidas a la esencia de la disciplina?

La respuesta a las preguntas de Klafki es, sin duda, el Conocimiento Didáctico del Contenido específico que se desea articular en una clase determinada frente a un conjunto específico de estudiantes.

Recientemente, Loughran, Mulhall y Berry (2004) han presentado dos herramientas para documentar el CDC de los profesores:

- CoRe (Content Representation, ReCo en español, por "Representación del Contenido").

- PaP-eRs (Professional and Pedagogical experience Repertoires, o RePyPs en español, por "Repertorios de experiencia profesional y pedagógica”).
La ReCo es una matriz en cuya primera fila aparecen las ideas centrales para impartir el tema que han sido declaradas por el profesor y en las otras filas cada una de ocho preguntas, con las que se logran documentar las ideas centrales para la enseñanza; los objetivos de la misma declarados por el profesor; su conocimiento de las concepciones alternativas de los alumnos y las dificultades de aprendizaje; la secuenciación apropiada de los tópicos; el empleo correcto de analogías, metáforas y ejemplos; las formas de abordar el entramado de ideas centrales; los experimentos, problemas y proyectos que el profesor emplea durante su clase; y las formas ingeniosas de evaluar el entendimiento que emplea, entre otras cuestiones.

En un trabajo muy reciente, Park, Jang, Che, \& Jung (2011) insisten en que la reforma educativa hacia un enfoque constructivista de la enseñanza - gracias al cual el aprendiz logre construir el conocimiento con base en lo que sabe y cree previamente, junto con las nuevas ideas que el profesor le pone en contacto- sólo es posible si el profesor entiende el nivel de comprensión del alumno y entonces extiende su diseño de instrucción para retar sus ideas previas. De esta forma, el profesor debe transformar el conocimiento del contenido en formas que sea pedagógicamente más poderosas, adaptadas a las variantes de las ideas previas, a los diferentes niveles de comprensión y dificultades de aprendizaje de los estudiantes individuales (el CDC, diría Shulman en pocas palabras). Formas de conocimiento que les permitan "seleccionar contenidos científicos y adaptarlos y diseñar 
currículos que atiendan los intereses, conocimientos, entendimientos, habilidades y experiencias de los estudiantes" (NRC, 1996, p. 30).

He seleccionado para mis investigaciones sobre CDC el contenido de la Química. Ya mostré en una investigación resumen en Garritz et al. (2008) algunos de los resultados obtenidos en la documentación del CDC en maestros del bachillerato $y$ en profesores universitarios, principalmente sobre la "estructura corpuscular de la materia”, y sobre los conceptos de "reacción química" y "cantidad de sustancia" (incluida su unidad, el mol); los tres temas pertenecen al corazón de la enseñanza básica de la química. Pero en esta ocasión vamos a hablar de otros tres tópicos de importancia, en este orden:

- CDC y la afectividad (Garritz, 2010);

- CDC de la estequiometría (Padilla \& Garritz, 2011);

- CDC de la indagación (Espinosa, Labastida, Padilla \& Garritz, 2011).

\section{CDC y la afectividad}

Hablemos para empezar de los buenos profesores y de sus aspectos afectivos. Hay varias citas para hacerlo, por ejemplo, Tobin y Fraser (1990) nos dicen que los profesores ejemplares (exemplary, en el original en inglés) de ciencia, además de otras tres cuestiones: "Mantienen un ambiente de aprendizaje favorable en el aula”. Once años más tarde, Brophy (2001) incluye en la primera de sus doce guías de la buena enseñanza basadas en la investigación: "Clima del aula de apoyo. Los estudiantes aprenden mejor dentro de comunidades de aprendizaje cohesivas y afectuosas".

Eso del 'clima' o 'el ambiente favorable' en las aulas debe formar parte del CDC, aunque la porción afectiva no se haya marcado explícitamente como integrante del mismo, sino acaso del conocimiento pedagógico general. No obstante, como hay una parte del contenido afectivo que tiene que ver con el contenido disciplinario, seguramente tiene relación con el CDC; al menos eso es lo que postulé recientemente (Garritz, 2010). Por ejemplo, el odio por el aprendizaje hacia las matemáticas o la química, el cual tiene mucho que ver con la complejidad de estas disciplinas. Otro par de autores (Park \& Oliver, 2008) consideran los cinco elementos tradicionales del CDC contemplados por Magnusson, Krajcik \& Borko (1999):

- Visión y propósito de la enseñanza de la ciencia;

- Conocimiento y creencias sobre el currículo de ciencia;

- Conocimiento y creencias acerca del entendimiento estudiantil sobre tópicos específicos de ciencia;

- Conocimiento y creencias sobre estrategias instruccionales para enseñar ciencia;

- Conocimiento y creencias sobre evaluación en ciencia.

pero agregan un sexto elemento de carácter afectivo, que denominan:

- Eficacia del profesor, la cual se refiere a la creencia que tiene el profesor de su capacidad para mejorar los resultados estudiantiles. Es sumamente importante el papel del profesor en definir las tareas a enseñar y organizar el conocimiento relevante hacia esas tareas. En este proceso esta forma más "afectiva" o provocativa de conocimiento juega un papel crucial. 
Así, desde hace casi veinte años, la porción afectiva del aprendizaje de las matemáticas (McLeod, 1992) engloba creencias, actitudes y emociones. Dentro de las dos primeras hay una gran cantidad de cosas que tiene que ver con el campo disciplinario (en los puntos redondos que siguen el autor ha sustituido "campo disciplinario" por "matemática”, como aparece en el original de McLeod), tales como:

- Creencias sobre la naturaleza del campo disciplinario y su aprendizaje.

- Creencias sobre uno mismo como aprendiz del campo disciplinario.

- Creencias sobre la enseñanza del campo disciplinario.

- La componente cognitiva de la actitud tiene que ver con expectativas y preferencias por el campo disciplinario y

- Su componente afectiva tiene que ver con sentimientos, emociones y estados de ánimo provocados por la disciplina (y varían de una disciplina a otra con mucha facilidad).

En relación también con la buena docencia, escribí hace poco (Garritz, Rueda \& Robles, 2011) que un buen profesor puede lograr el objetivo de la educación científica, o sea, "el desarrollo de individuos autónomos que empleen el razonamiento científico como norma, que revelen un conocimiento sólido acerca de la tecnología y que tengan conciencia del impacto de la ciencia y la técnica sobre la sociedad, capaces de pensar por sí mismos, de tomar decisiones, confiados en su capacidad para enfrentar lo nuevo y asumir la responsabilidad ética de sus acciones, tanto en el ámbito individual como en el profesional y ciudadano". Todo ello debe lograrlo con comprensión, respeto y afectividad, intentando provocar la motivación y la emoción en sus estudiantes.

Algunos resultados recientes sobre un nuevo cuestionario en el que hemos incluido algunas preguntas sobre afectividad, dirigido a cuatro profesores de Química General (Ortega-Villar \& Garritz, en elaboración) son por ejemplo, de carácter general las siguientes:

- ¿Cómo logra la emoción de los estudiantes por el tema?

- ¿Cómo promueve actitudes favorables hacia el aprendizaje de este tema?

- ¿Cómo favorece que los alumnos participen y se expresen con confianza en el aprendizaje del tema?

Y para cada uno de los conceptos centrales discutidos con los profesores en la enseñanza del enlace químico en materia condensada (Propiedades físicas; Parámetros de Enlace: Energía y Distancia; Polaridad e Interacción Intermolecular; Redes [Metálica, iónica o covalente]) se plantean estas otras:

- ¿De qué manera promueve la motivación estudiantil para el aprendizaje de este concepto? Indique el tipo de motivación al que recurre.

- ¿De qué manera promueve el interés individual y tópico del alumno para el aprendizaje de este concepto? ¿Hace uso de anécdotas, alegorías, metáforas, cuentos, preguntas, contradicciones u otros recursos? Explíquelos. 


\section{CDC de la estequiometría}

La estequiometría, o ciencia "de los cálculos químicos”, ha jugado un papel clave en la evolución de la química como ciencia. Como lo indica Kolb (1978) el término viene del griego stoicheion (elemento) y metron (medida). Fue desarrollada por el químico alemán Jeremias Benjamin Richter (17621807), como una manera de cuantificar las masas de las sustancias que se combinan en una reacción química. Richter encontró que las proporciones de las masas de los reactivos eran constantes, por ejemplo, las cantidades equivalentes de un ácido y una base en una reacción de neutralización. Richter era un matemático interesado en la química que pensaba que ésta podría considerarse como una rama de las matemáticas.

Los participantes en este estudio fueron cuatro profesores universitarios de México y Argentina. Uno de ellos tiene 15 años de experiencia y obtuvo su doctorado en Química Inorgánica, con posdoctorado en una renombrada universidad de la Gran Bretaña. Otros dos son licenciados en Ingeniería Química con más de treinta años de experiencia docente. El cuarto es un profesor con doctorado en Bioquímica y casi 30 años de experiencia docente. Todos ellos son considerados como magníficos profesores por sus pares y sus estudiantes, además de tener libros escritos sobre el tema.

Encontramos en esta investigación que hay profesores que utilizan la estequiometría en la enseñanza con un objetivo fundamentalmente algorítmico (en la búsqueda de procedimientos, sin preguntarse mucho acerca de la comprensión profunda de lo que se hace y por qué se hace). Sin embargo, otros profesores lo emplean para reforzar algunos conceptos cruciales del concepto de reacción química o inclusive sobre la constitución corpuscular de la materia.

Concluimos que podrían existir cuatro enfoques pedagógicos que caracterizamos como:

- Conceptual (la construcción de una visión holística del contenido por un pensamiento crítico inductivo $\mathrm{y}$ deductivo).

- Representacional (el uso de diferentes tipos de representaciones para mejorar el aprendizaje, tales como históricas, analógicas, visuales, mapas, experimentos, demostraciones y modelos).

- Contextual (la contextualización puede ayudar a los estudiantes a pensar críticamente y para darse cuenta de la relevancia de la química en su vida diaria).

- Procedimental (el conocimiento que requiere el empleo de un conjunto de procedimientos memorizados para la solución de un problema, lo que denota el uso dinámico y exitoso de ciertas reglas o algoritmos con formatos de representación relevantes).

De esta manera, llevamos a cabo un análisis equivalente al desarrollado en el tema de cantidad de sustancia (Padilla et al., 2008) en que contamos cada una de las frases expresadas por el profesor en su ReCo clasificándolas en zonas del perfil conceptual de Mortimer (1995). El resultado de este conteo se presenta en la ilustración 1. 


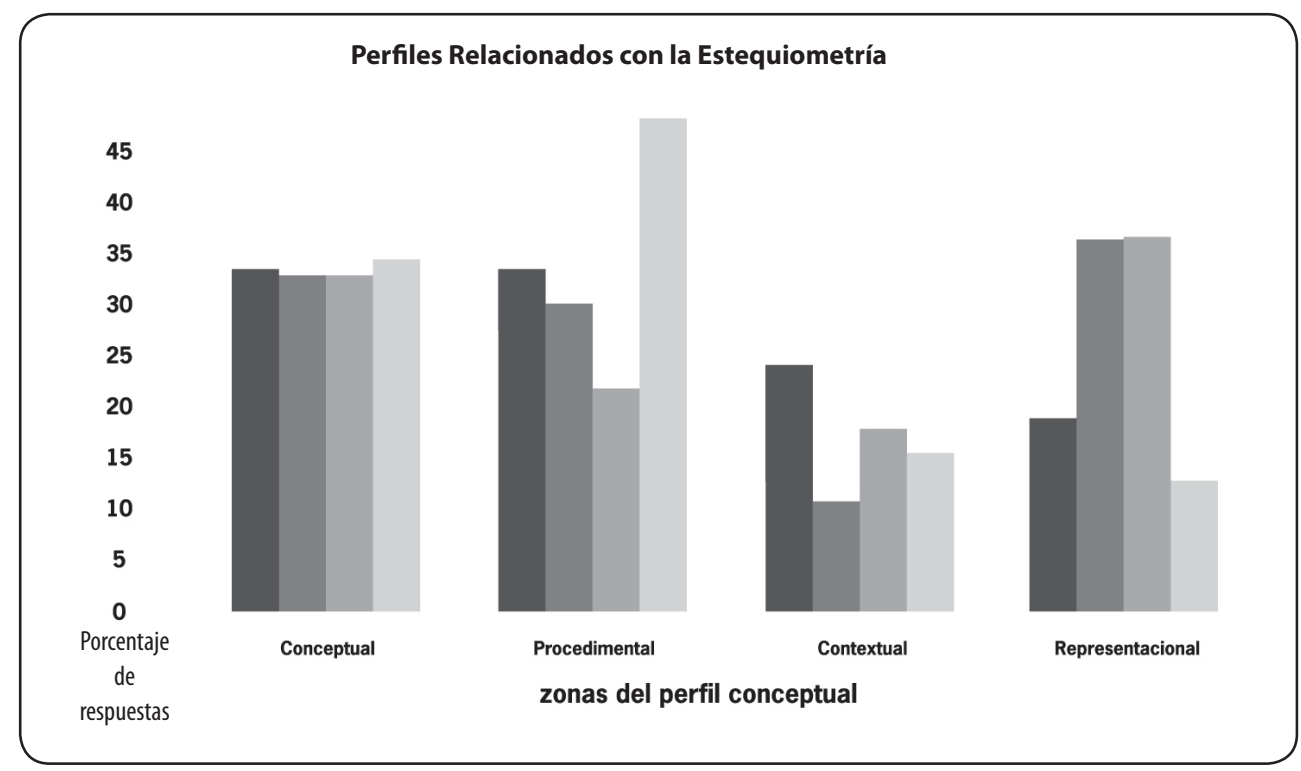

Ilustración 1. Porcentaje de respuestas en cada una de las cuatro zonas de perfil conceptual elegidas: Conceptual, Representacional, Contextual y Procedimental.

Podemos ver en todos los profesores alrededor de un 30\% de frases en la zona conceptual, pero el resto de las zonas muestra diferente predominancia en cada uno de los cuatro profesores: Los profesores 2 y 3 son preferentemente representacionales; aunque uno de ellos lo es por el amplio uso de analogías y el otro por el empleo de narrativas históricas; El profesor 4 es el más procedimental de los cuatro y; El profesor 1 tiene una mezcla algo equilibrada de las otras tres zonas.

En este trabajo analizamos (Padilla \& Garritz, 2011) que el aprendizaje conceptual contra el procedimental se considera una supuesta dicotomía inconveniente de promover. Existen varios estudios que indican que los alumnos tienen por lo general más éxito en resolver problemas mediante el uso de un procedimiento memorizado que otros con una base mucho más conceptual (Bunce, 1993; Nakhleh, 1993). Llegamos a la conclusión de que resulta sumamente conveniente combinar ambas cuestiones (la procedimental y la conceptual) para que no se dé una dicotomía como la representada por los siguientes pares de palabras:

- Conceptual vs práctica;

- Saber qué vs saber cómo;

- Declarativo vs procedimental;

- Hechos vs habilidades;

- Comprensión vs algorítmico;

- Teológica vs esquemática;

- Deductiva vs empírica;

- Significativa vs mecánica;

- Lógica/relacional vs instrumental;

- Estructural vs operacional

Recomendamos de esta forma que el profesor se aboque a combinar los enfoques conceptual y procedimental, o es más, inclusive también los otros dos enfoques, contextual y representacional, 
ya que en la educación es importante fomentar las habilidades de pensamiento y la multi-modalidad cerebral, para que el alumno pueda responder a las siguientes cuatro preguntas: ¿yo sé qué? (conceptual) ¿yo sé por qué? (contextual y representacional), ¿yo sé cómo? (procedimental) y ¿yo sé cómo sé? (metacognitiva). Cualquier intento por hacer énfasis en una sola de estas cuestiones implica una enseñanza basada en el comportamiento ("behaviorismo" de Skinner). Debemos tomar en consideración la presencia de todos estos tipos de conocimiento mientras se aprende, como algo similar a la complementariedad fomentada en las culturas Mesoamericanas y de Oriente.

\section{CDC de la indagación}

En los Estándares Nacionales de la Educación Científica en los Estados Unidos se hace referencia a la indagación como parte fundamental del proceso de enseñanza / aprendizaje:

- La indagación científica se refiere a las diversas formas en las cuales los científicos abordan el conocimiento de la naturaleza y proponen explicaciones basadas en la evidencia derivada de su trabajo. La indagación también se refiere a las actividades estudiantiles en las cuales desarrollan conocimiento y entendimiento de ideas científicas...

- La indagación es una actividad polifacética que implica hacer observaciones; plantear preguntas; examinar libros y otras fuentes de información para ver qué es lo ya conocido; planificar investigaciones; revisar lo conocido hoy en día a la luz de las pruebas experimentales; utilizar instrumentos para reunir, analizar e interpretar datos; proponer respuestas, explicaciones y predicciones; y comunicar los resultados (NRC, 1996; p. 23).

Para la enseñanza de la ciencia, el CDC debe incluir, enfáticamente, el entendimiento de la indagación como una aproximación al contenido (Eick 2000). Planear y llevar a cabo un aprendizaje basado en la indagación en el aula es una tarea muy demandante para los profesores, ya que requiere de todo un conjunto de conocimientos: del contenido, pedagógico, de la indagación y de cómo implementarla. Ahora que un conocimiento teórico de todo ello tampoco le lleva a ninguna parte, pues debe emplearlos todos ellos de una manera integrada en el aula (Schuster \& Cobern, 2011), lo cual sólo es posible con el ejercicio práctico o, como dice Shulman (1987) con un modelo de razonamiento y acción pedagógicos.

En esta investigación (Espinosa, Labastida, Padilla \& Garritz, 2011) hemos presentado varias actividades propias de la indagación, a partir de una serie de referencias que tocan el punto (Schwab, 1978; NRC, 1996; 2000; Bybee, 2004; Lederman, 2004; and Khan, 2007). El resultado ha sido el de las siguientes siete actividades de la indagación:

- Identificar y plantear preguntas que puedan ser respondidas mediante la indagación;

- Definir y analizar bien el problema a resolver e identificar sus aspectos relevantes;

- Reunir información bibliográfica para que sirva de prueba;

- Formular explicaciones al problema planteado, a partir de las pruebas;

- Plantear problemas de la vida 
cotidiana y tocar aspectos históricos relevantes;

- Diseñar y conducir trabajo de investigación a través de diversas acciones (Reflexionar sobre las observaciones y fomentar la búsqueda de patrones en la información; Generar relaciones hipotéticas y evidencias entre las variables; Postular factores causales potenciales; Evaluar la consistencia empírica de la información; Hacer uso de analogías y/o de la intuición para ayudar a conceptualizar los fenómenos; Formular y manipular modelos físicos y mentales; Utilizar herramientas apropiadas y técnicas para reunir, analizar e interpretar datos; Pensar crítica y lógicamente para desarrollar predicciones, explicaciones, y modelos empleando las evidencias; Coordinar los modelos teóricos con la información; Evaluar las explicaciones alcanzadas, con algún modelo científico; Comunicar hechos y procedimientos científicos en la clase);

- Compartir con otros mediante argumentación lo que ha sido aprendido a través de la indagación.

Estas siete actividades se han colocado en el marco llamado "Representación del Contenido" (ReCo) de Loughran, Mulhall y Berry (2004), llevando a cabo cinco preguntas sobre cada una de estas actividades a cuatro profesores mexicanos de química del bachillerato (tres de la Escuela Nacional Preparatoria y uno del Instituto de Educación Media Superior del Distrito Federal) y uno que enseña física en la universidad (la Autónoma de la Ciudad de México), con el objetivo de recabar los datos sustanciales sobre cómo abordan la enseñanza a través de la indagación en el aula o el laboratorio. Esas cinco preguntas, tomadas del ReCo original de Loughran fueron:

- Describa brevemente porqué considera importante el desarrollo de algunas de las actividades de indagación mencionadas (Responda usted solamente para aquellas que utiliza en su labor docente tanto esta pregunta como las cuatro que siguen) - ¿A qué dificultades o limitaciones se enfrenta al implantar esta actividad? - ¿Cuáles considera que son las dificultades de los estudiantes cuando aborda esta actividad?

- ¿Qué ejemplos y procedimientos emplea para llevar a cabo esta actividad?

- ¿De qué manera evalúa si los estudiantes han entendido o se han confundido con esta actividad?

Aplicar las siete actividades mencionadas de la indagación para resolver un problema determinado es algo necesario para que los alumnos entiendan y evalúen las explicaciones que aparecen en periódicos y revistas, y es importante que valoren su credibilidad y validez (McNeill \& Krajcik, 2008).

Nuestros resultados señalan como respuesta de los profesores encuestados a la primera pregunta que la indagación es considerada como una actividad primordial para su modo de enseñar. Incluimos aquí las frases textuales de dos de ellos al respecto: 
Considero que la indagación constante en el salón de clase, en el laboratorio y en su vida cotidiana es muy formativa y estimulante para los alumnos; además, ayuda a desarrollar en ellos el hábito de observar, de razonar y de relacionar diferentes conceptos tanto de la propia asignatura como de otras y de su experiencia personal (profesor 1).

Con base en algunos estudios hechos desde la perspectiva piagetiana, buena parte de los estudiantes de los primeros semestres de licenciatura se encuentra entre el periodo concreto y el formal. Para que ocurra la transición es necesario que haya un involucramiento mental activo por parte del aprendiz, por lo cual - mi grupo de trabajo y yo-consideramos que la "indagación guiada en el contexto del laboratorio" (o aprendizaje como investigación orientada, como lo llaman otros; Gil, 1983), es la estrategia pedagógica adecuada para los estudiantes en esta situación (profesor 2).

Solamente dos profesores contestaron positivamente a la actividad de 'formular explicaciones a partir de la evidencia' y marcaron su relevancia.

Esta es la parte principal de la finalidad de la indagación y considero que es la forma de adquirir un conocimiento y no aprender de memoria definiciones de conceptos (Profesor 1).

Es un elemento fundamental el formular explicaciones a un problema a partir de la evidencia. El ejercicio obliga a analizar la información, establecer relaciones, echar mano del bagaje de conocimientos con los que se cuenta, incorporar la información recabada en la investigación bibliográfica, organizar todos los datos y generar una explicación plausible. (Profesor 3).
Resulta importante relacionar el conocimiento científico con los hechos de la vida cotidiana y con acontecimientos históricos. En relación con aspectos de este tipo, hemos seleccionado tres frases de los profesores (dos de ellos sobre aspectos de la vida diaria y el tercero sobre aspectos históricos):

La mayor dificultad a la que se enfrenta uno es precisamente la búsqueda, o diseño, o selección del problema que plantee una situación de la vida cotidiana creíble, acorde con la realidad del estudiante, que sea a la vez de fácil comprensión para él, pero que al mismo tiempo tenga un grado de dificultad que vuelva atractiva la búsqueda de la resolución (Profesor 5).

Porque el problema a abordar debe ser lo más cercano posible a la cotidianidad del estudiante, para así facilitar el aprendizaje significativo. Además, el estudiante tiene que darse cuenta de que la construcción de la ciencia es un proceso histórico (Profesor 4).

Es importante el momento histórico en el que se desenvuelven los hallazgos científicos y la construcción del conocimiento científico no debe mostrarse como un evento aislado y completamente ausente de la realidad del momento, sino como parte de su momento, susceptible a la influencia de éste y como resultado de las propias condiciones del mismo (Profesor 1).

Cuatro de los profesores marcaron la actividad de 'hacer investigación' que ellos desarrollan durante sus cursos. Aquí ponemos lo que indicó uno de ellos: 
Por ejemplo, poder contestar a la pregunta ¿qué necesito saber para poder predecir si cierto objeto se hunde oflota en un líquido dado? implica aprender a definir operacionalmente las propiedades y luego aprender a hacer experimentos donde se controlen las variables e investigar si cada una ellas influye o determina el comportamiento de hundimiento o flotación del objeto (Profesor 2).

Entre las conclusiones a las que llegamos en este trabajo podemos recalcar el gran interés que tienen estos profesores por mejorar la enseñanza tradicional de asignaturas como física y química, pues están convencidos de que el proceso de indagación conlleva a trascender de una enseñanza meramente informativa hacia una enseñanza más dinámica, basada en la reflexión constante derivada del planteamiento de preguntas contextualizadas, y que para dar con una respuesta plausible es necesario proponer un diseño lógicamente estructurado que permita dar una respuesta basada en las pruebas de los planteamientos. Coinciden, aunque sea parcialmente, en lo importante que resulta el planteamiento de preguntas, la búsqueda de información en la bibliografía y la realización de investigaciones. Todos los profesores están de acuerdo en que la indagación conduce a los estudiantes hacia la modificación de su forma de pensar, tornándola más científica.

Con la indagación dirigida y contextualizada se logra que los estudiantes poco a poco establezcan relaciones y razonamientos cercanos a los de los científicos, es decir, cómo utilizar actividades de pensamiento crítico. A pesar de que la indagación no forma parte integral del currículo en muchos de nuestros países, estos cinco profesores la emplean con éxito en sus clases.

\section{Conclusiones}

Cierro este trabajo con una disculpa por haber tratado temas tan disímbolos en él, aunque todos ellos tengan que ver con el conocimiento didáctico del contenido. Uno de ellos, el de la estequiometría, lo es trasparentemente, por ser un tema del curso de Química General Universitaria en el que identificamos cuatro formas epistemológicas de tratarlo, pero desembocamos en la conclusión del valor que tiene su tratamiento combinado; otro, el de la afectividad, todavía se discute inclusive si forma parte del CDC o no, aunque yo defiendo que sí, que hay aspectos del contenido relacionados con lo afectivo (la motivación, las metas, las auto-creencias como la auto-estima, el auto-concepto, la auto-eficacia, etc.); el tercero, el de la indagación Lederman (2004) insiste en que, al igual que la Naturaleza de la Ciencia, debe formar parte explícita del conocimiento de los profesores, como si fuera un tema disciplinario más; insistir sobre los alumnos para que reflexionen en los procedimientos que siguieron, porqué lo hicieron y qué implicaciones tiene eso sobre el conocimiento producido. La indagación debe desembocar el algo después de una planeación, su enseñanza explícita y su evaluación sistemática.

Es de tal variedad el conocimiento base de un profesor, es de tal amplitud el conocimiento didáctico del contenido dentro de aquel, tienen tales interrelaciones todos sus aspectos, que resulta una tarea sumamente compeja sistematizar y documentar el CDC de algunos profesores ejemplares, lo cual puede ser de gran ayuda en el proceso formativo 
de nuevos profesores. Simplemente, discutir en un grupo de profesores en formación la ReCo de un profesor

\section{Bibliografía}

Bybee, R. (2004). Scientific Inquiry and Science Teaching. En Flick, Lawrence B. and Norman G. Lederman (eds.) Scientific Inquiry and Nature of Science, (Chapter 1, pp. 1-14), Dordrecht, The Netherlands: Kluwer Academic Publishers.

Brophy, J. (2001). Introduction. Generic Guidelines for Good Teaching. In J. Brophy (Ed.) Subject-specific instructional methods and activities, (Pp. 1-23). Advances in Research on teaching, vol 8. Amsterdam: JAI.

Bunce, D. M. (1993). Introduction: Symposium: Lecture and learning: Are they compatible? Journal of Chemical Education, 70(3), 179-180.

Eick, C. J. (2000). Inquiry, nature of science, and evolution: The need for a more complex pedagogical content knowledge in science teaching. Electronic Journal of Science Education, 4 (3).

Espinosa-Bueno, J. S., Labastida-Piña, D. V., Padilla, K. y Garritz, A. (2011). Pedagogical Content Knowledge of Inquiry: An Instrument to Assess It and Its Application to High School In-Service Science Teachers, US-China Education Review, 8(3), $i$ ?- $i$ ? In the press.

Garritz, A., Nieto, E., Padilla, K., Reyes, F. y Trinidad, R. (2008). Conocimiento didáctico del contenido en química. Lo que todo profesor debería poseer. Campo Abierto, 27(1) 153-177. Publicación de la Facultad de Educación de la Universidad de Extremadura, España. extraordinario, puede darles sin duda una serie de buenas ideas para encarar la enseñanza de un tema en específico.

Garritz, A. (2010). Pedagogical Content Knowledge and the affective domain of Scholarship of Teaching and Learning, International Journal for the Scholarship of Teaching and Learning, 4(2), 1-6. Puede consultarse en la URL http://academics.georgiasouthern. edu/ijsotl/v4n2/personal_reflections/_Garritz/index.html

Garritz, A.; Rueda, C. \& Robles, C. (2011). Opiniones de profesores y estudiantes mexicanos del bachillerato y la universidad públicos sobre la naturaleza de la ciencia y la tecnología: una pobreza alarmante. En: A. Bennàssar-Roig, Á. Vázquez-Alonso, M. A. Manassero-Mas \& A. García-Carmona (coords.), Ciencia, tecnología y sociedad en Iberoamérica: Una evaluación de la comprensión de la naturaleza de ciencia y tecnología, capítulo 8, (Pp. 115-125), Madrid: Organización de Estados Iberoamericanos (OEI). El libro completo puede bajarse en la URL http://www.oei.es/salactsi/ DOCUMENTO5vf.pdf

Khan, S. (2007), Model-Based Inquiries in Chemistry, Science Education, 91, pp. 877 - 905.

Lederman, N. (2004), Syntax of nature of science within inquiry and science instruction, Chapter 14. En Flick, L. \& Lederman, N., (eds.) Scientific Inquiry and Nature of Science, pp. 301-317, Dordrecht, The Netherlands: Kluwer Academic Publishers.

Klafki, W. (1958). Didaktische Analyse als Kern der Unterrichtsvorbereitung. Basel: Wienheim. 
Loughran, J., Mulhall, P. \& Berry, A. (2004). In Search of Pedagogical Content Knowledge in Science: Developing Ways of Articulating and Documenting Professional Practice, Journal of Research in Science Teaching, 41(4), 370-391.

Magnusson, S., Krajcik, L., \& Borko, H. (1999). Nature, sources and development of pedagogical content knowledge. En J. Gess-Newsome \& N. G. Lederman (Eds.), Examining pedagogical content knowledge. The Construct and its Implications for Science Education. (pp. 95-132). Dordrecht, The Netherlands: Kluwer.

McLeod, D. B. (1992). "Research on affect in mathematics education: A reconceptualization". En D. A. Grouws (Ed.), Handbook of Research on Mathematics Teaching and Learning (pp.575598). New York: Macmillan.

McNeill, K. L. \& Krajcik, J. (2008). Inquiry and scientific explanations: Helping students use evidence and reasoning. In Luft, J., Bell, R. \& Gess-Newsome, J. (Eds.). Science as inquiry in the secondary setting. (p. 121-134). Arlington, VA: National Science Teachers Association Press. Puede bajarse de la siguiente página de la autora http://www.katherinelmcneill.com/ uploads/1/6/8/7/1687518/mcneillkrajcik_nsta_inquiry_2008.pdf

Mortimer, E.F. (1995). Conceptual change or conceptual profile change? Science \& Education, 4, 267-285.

Nakhleh, M. B. (1993). Are Our Students Conceptual Thinkers or Algorithmic Problem Solvers? Identifying Conceptual Students in General Chemistry, Journal of Chemical Education, 70(1), $52-5$.

NRC “National Research Council”. (1996). National science education standards.
Washington, DC: National Academy Press.

NRC “National Research Council” (2000). Inquiry and the National Science Education Standards. Washington, DC: National Academies Press.

Nurrenbem, S. C. \& Pickering, M. (1987). Concept Learning versus Problem Solving: Is there a difference?, Journal of Chemical Education, 64(6), 508-510.

Ortega-Villar, N. \& Garritz, A. (en elaboración) Research on the affective domain with examples of its relationship with the subject matter content.

Padilla, K. \& Garritz, A. (2011). The Pedagogical Content Knowledge of university chemistry professors teaching stoichiometry, 20 pages. Proceedings of the NARST-2011 Conference, Orlando, FL, USA, April 3rd to 6th.

Padilla, K., Ponce-de-León, A. M., Rembado, F. M. \& Garritz, A. (2008). Undergraduate Professors' Pedagogical Content Knowledge: The case of 'amount of substance', International Journal of Science Education, 30(10), 1389-1404.

Park, S., \& Oliver, J. S. (2008). Revisiting the conceptualisation of pedagogical content knowledge (PCK): PCK as a conceptual tool to understand teachers as professionals. Research in Science Education, 38(3), 261-284.

Park, S.; Jang, J. Y.; Chen, Y. C. \& Jung J. (2011) . Is Pedagogical Content Knowledge (PCK) Necessary for Reformed Science Teaching? Evidence from an Empirical Study, Research in Science Education, 41(2), 245-260.

Pickering, M. (1990). Further Studies on Concept Learning versus Problem Solving, Journal of Chemical Education, 67(3), 254-5. 
Posner, G. J., Strike, K. A., Hewson, P. W. \& Gertzog, W. A. (1982). Accommodation of a Scientific Conception: Toward a Theory of Conceptual Change, Science Education, 66(2), 211-227.

Schuster, D. \& Cobern, W. W. (2011). Assessing Pedagogical Content Knowledge of inquiry science instruction, 12 pages. Proceedings of the NARST-2011 Conference, Orlando, FL, USA, April 3rd to 6 th.

Schwab, Joseph (1978), Science, curriculum and liberal education, Chicago:University of Chicago Press.

Shulman, L. S. (1987). Knowledge and Teaching: Foundations of the New Reform, Harvard Educational Review, 57(1), 1-22. Traducido al español como "Conocimiento y enseñanza: fundamentos de la nueva reforma", Profesorado. Revista de currículum y formación del profesorado, 9, 2 (2005). Puede bajarse de la siguiente página de Redalyc: http://redalyc. uaemex.mx/src/inicio/ArtPdfRed. jsp?iCve $=56790202$

Shulman, L. S. (1999). "Foreward". En Gess-Newsome, J., Lederman, N. G. (Eds.), Examining Pedagogical Content Knowledge. The Construct and its Implications for Science Education. (Pp. ix-xii) Dordrecht, The Netherlands: Kluwer Academic Publishers.

Tobin, K. \& Fraser, B. J. (1990) What does it mean to be an exemplary science teacher? Journal of Research in Science Teaching, 27, 3-25. 


\section{THEA}
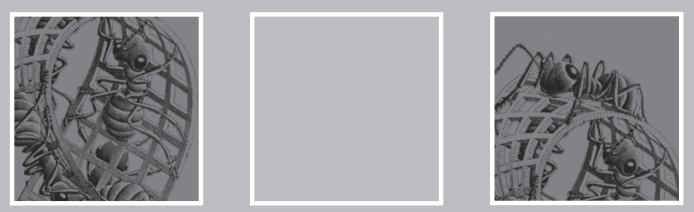

Artículos de

\section{Ensayo}

UNIVERSIDAD PEDAGOGICA NACIONAL

Educadora de educadores

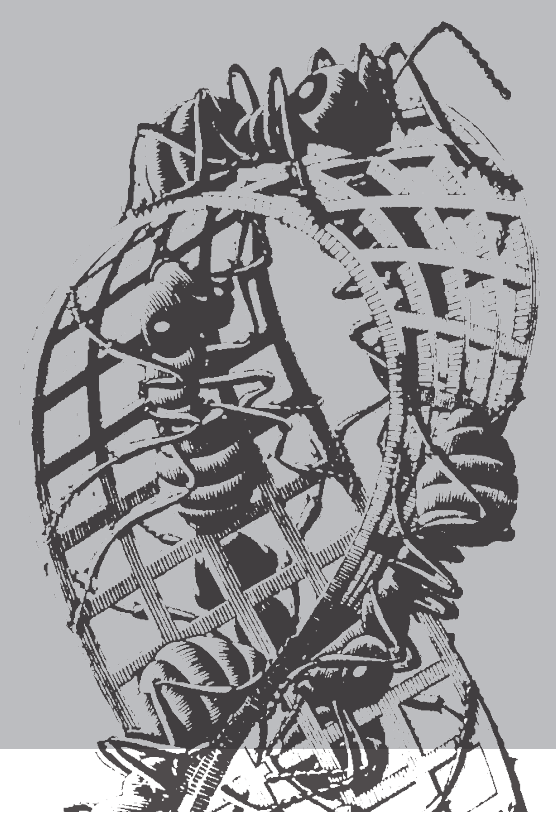

